\title{
Superspace approach to high pressure superstructures
}

Article in High Pressure Research · August 2013

DOI: 10.1080/08957959.2013.822076

5 authors, including:

Maxim Bykov

University of Bayreuth

83 PUBLICATIONS 216 CITATIONS

SEE PROFILE

Michael Hanfland

European Synchrotron Radiation Facility

428 PUBLICATIONS 12,494 CITATIONS

SEE PROFILE
Elena Bykova

University of Bayreuth

127 PUBLICATIONS 614 CITATIONS

SEE PROFILE

Hanns-Peter Liermann

Deutsches Elektronen-Synchrotron

183 PUBLICATIONS 2,123 CITATIONS

SEE PROFILE

Some of the authors of this publication are also working on these related projects:

Dynamic loading and unloading of $\mathrm{SiO} 2$ aggregates. Real-time phase transformations monitored by means of synchrotron beam diffraction View project

High-pressure synthesis of polynitrides View project 
This is an Author's Original Manuscript of an article submitted for consideration in the High Pressure Research (copyright Taylor \& Francis); available online at http: //www.tandfonline.com/10.1080/08957959.2013.822076 


\title{
Superspace approach to high-pressure superstructures
}

\author{
Maxim Bykova $^{\mathrm{a}}$, Elena Bykova ${ }^{\mathrm{a}, \mathrm{b}}$, Michael Hanfland ${ }^{\mathrm{c}}$, Hanns-Peter Liermann ${ }^{\mathrm{d}}$ and Sander van \\ Smaalen $^{\mathrm{a} *}$ \\ ${ }^{a}$ Laboratory of Crystallography, University of Bayreuth, 95440 Bayreuth, Germany \\ ${ }^{\mathrm{b}}$ Bayerisches Geoinstitut, Universität Bayreuth, 95440 Bayreuth, Germany \\ ${ }^{\mathrm{c}}$ European Synchrotron Radiation Facility (ESRF), 38043 Grenoble, France \\ dPhoton Sciences, FS-PE, Deutsches Elektronen Synchrotron (DESY), 22607 Hamburg, \\ Germany
}

(Received 00 Month 20XX; final version received 00 Month 20XX)

\begin{abstract}
Single-crystal X-ray diffraction in a diamond anvil cell has proven to be a powerful tool for studying matter at extreme conditions. Displacive zone-boundary phase transitions between a high-symmetry low-pressure structure and a low-symmetry high-pressure superstructure represent a significant part of pressure-induced processes. However, analysis of such superstructures is usually complicated by low angular resolution and limited reciprocal space coverage. This leads to unfavorable data/parameter ratio during the structure solution and refinement. The superspace approach is an established method to describe commensurately modulated structures, however it is not often applied to high-pressure phase transitions. Here we discuss the advantages of the superspace approach for the description and crystal-chemical analysis of high-pressure superstructures. The main ideas of this approach are illustrated on the examples of isostructural $\mathrm{FeOCl}$ and $\mathrm{CrOCl}$, forming different superstructures above $15 \mathrm{GPa}$.
\end{abstract}

Keywords: phase transitions, high-pressure single-crystal x-ray diffraction, superspace approach.

\section{Introduction}

X-ray diffraction in a diamond-anvil cell (DAC) is a powerful tool for studying materials at high pressures [1, 2]. The application of external pressure leads to various phenomena including insulator-metal transitions [3, 4, high-spin to low-spin transitions [5], enhancement of superconductivity [6], and normal-to-incommensurate phase transitions in elemental metals [7. A group of phase transitions between a high-symmetry form, and a low-symmetry distorted form stable at higher pressures represent a significant part of pressure-induced processes [8 11].

High-pressure X-ray diffraction usually suffers from undesirable diffraction from diamonds, gasket and pressure-transmitting medium, insufficient data resolution and low coverage of the reciprocal space. To increase the coverage, several crystals in different orientations may be placed into a DAC. However sometimes the morphology of the crystal, e.g. in case of plate-like crystals predefines its orientation. In this sense, the conventional analysis of complex superstructures may be quite difficult due to the very small ratio between the number of independent reflections and the number of refined parameters.

\footnotetext{
*Corresponding author. Email: smash@uni-bayreuth.de
} 
The superspace approach is an established method to describe incommensurate and commensurate superstructures $[12$ 14. Furthermore, in the last years a number of computer programs were developed for convenient data processing, structure solution and refinement. Among them are SUPERFLIP [15], the program for the structure solution in arbitrary dimensions, JANA2006 computing system for structure refinements and analysis [16], and EVAL15 integration software [17].

The main idea behind the superspace description is that the superstructure may be interpreted as its basic structure with three-dimensional space group symmetry and a small unit cell, which is subjected to a periodic deformation. Modulation functions are characterized by a wave vector $\boldsymbol{q}$, whose components are given with respect to the basis vectors of the reciprocal lattice of the basic structure:

$$
\boldsymbol{q}=\sigma_{1} \boldsymbol{a}^{*}+\sigma_{2} \boldsymbol{b}^{*}+\sigma_{3} \boldsymbol{c}^{*}
$$

Commensurate modulations are described by modulation wave vectors with only rationalvalued components. Therefore, an integer $N$ exists, for which $N \boldsymbol{q}$ is equal to reciprocal lattice vector of the basic structure.

Within the superspace approach, the position $(\boldsymbol{x})$ of the atom $\mu$ is defined by its position in the basic structure $(\overline{\boldsymbol{x}})$ plus a displacement:

$$
\boldsymbol{x}=\overline{\boldsymbol{x}}+\boldsymbol{u}^{\mu}\left(\bar{x}_{4}\right),
$$

where $\bar{x}_{4}=t+\boldsymbol{q} \cdot \overline{\boldsymbol{x}}$, with $t$ being the phase of modulation and $\boldsymbol{u}^{\mu}\left(\bar{x}_{4}\right)$ is the modulation function of atom $\mu$. In principle, the only requirement on the modulation functions is that they are periodic, so that $\boldsymbol{u}^{\mu}\left(\bar{x}_{4}\right)=\boldsymbol{u}^{\mu}\left(\bar{x}_{4}+1\right)$. However, it is convenient to represent atomic modulation functions as Fourier series:

$$
u_{i}^{\mu}\left(\bar{x}_{4}\right)=\sum_{n=1}^{n_{\max }} A_{i}^{n}(\mu) \sin \left(2 \pi n \bar{x}_{4}\right)+B_{i}^{n}(\mu) \cos \left(2 \pi n \bar{x}_{4}\right),
$$

for $i=x, y, z$. Therefore, for the structural analysis in superspace $A_{i}^{n}$ and $B_{i}^{n}$ are the parameters that must be determined along with the atomic coordinates in the basic structure.

In case of commensurate modulations, the model described in superspace might need less parameters than the supercell in three-dimensional (3D) space, because it is usually possible to concentrate on the most important parameters, like low-order harmonics in the Fourier expansions of the modulation functions, while higher-order modulation parameters may be not substantial for the distortion. This reduction in the number of independent parameters is especially important for highly incomplete high-pressure data. Despite the obvious usefulness of superspace description of superstructures, it is rarely applied by high-pressure crystallographers. One of the few examples is the high-pressure phase of $\mathrm{Ga}$, that seems to be rather complex at first sight but can be described with a simple distortion of the average structure using 4 modulation parameters instead of the 38 parameter needed when using a conventional approach [18. Another method to reduce the number of parameters is to use symmetry mode analysis and to refine the amplitudes of the most important modes only. However, especially in case of large superstructures a mode decomposition may be less efficient [19]. Here we will demonstrate the usefulness of the superspace approach on the examples of isostructural layered $\mathrm{FeOCl}$ and $\mathrm{CrOCl}$, forming different superstructures above $15 \mathrm{GPa}$. 


\section{High-pressure superstructures of transition metal oxychlorides}

\subsection{Experiment preparation and data collection}

The use of synchrotron facilities is a preferable choice for any high-pressure X-ray diffraction experiment. The high-energy of the synchrotron X-ray photons allows to significantly increase the angular resolution compared to conventional in-house sources. Small focus size allows to get rid of gasket diffraction and of strain gradients within the crystal. High intensity reduces data collection times and enables detection of weak superstructure reflections in case of small distortions. We have performed single-crystal X-ray diffraction experiments on the high-pressure phases of $\mathrm{FeOCl}$ and $\mathrm{CrOCl}$ at beamlines P02.2 (DESY, Hamburg) and ID09A (ESRF, Grenoble), respectively. The data collection for FeOCl was performed using a wavelength of $0.29004 \AA$ and a Mar345 image plate detector, while the data collection for $\mathrm{CrOCl}$ was performed using a wavelength of $0.4144 \AA$ and a Mar555 flat panel detector. For both experiments we have used the combination BX90 DACs [20] with Boehler-Almax anvils [21] which provide a large opening angle of $80^{\circ}$ in $4 \theta$. Such a large X-ray opening is extremely important for single-crystal X-ray diffraction, in order to increase reciprocal space coverage. Perfect quality single crystals of $\mathrm{FeOCl}$ and $\mathrm{CrOCl}$ were loaded in a $125 \mu \mathrm{m}$ hole of an preindented rhenium gasket along with ruby spheres for pressure determination [22]. The DACs were loaded with neon as pressure-transmitting medium using the BGI gas loading system [23. For each sample, 80 independent frames in an $\omega$-scan range of $-40^{\circ}$ to $+40^{\circ}$ were collected $\left(1^{\circ}\right.$ scanning step size) with an exposure time of one second.

\section{$2.2 \quad$ Indexing and integration of diffraction patterns}

$\mathrm{FeOCl}$ and $\mathrm{CrOCl}$ belong to a group of isostructural layered transition metal oxychlorides, $M \mathrm{OCl}(M=\mathrm{Ti}, \mathrm{V}, \mathrm{Cr}, \mathrm{Fe})$, which have recently been investigated due to their low-dimensional magnetic properties [24-27]. At ambient conditions $\mathrm{MOCl}$ possess the orthorhombic space group Pmmn. Their structure consists of double $M$-O layers, separated by $\mathrm{Cl}$ bilayers (Figure 1). Highly anisotropic compression of these compounds lead to structural instabilities at high pressure and they undergo a phase transition in the vicinity of $15 \mathrm{GPa}$, which is accompanied by an appearance of weak superlattice reflections. The diffraction pattern of $\mathrm{FeOCl}$ at $22.7 \mathrm{GPa}$ may be indexed with the orthorhombic lattice as valid for the low-pressure phase and a modulation wavevector $\boldsymbol{q}^{F e}$ $=\left(\frac{1}{4}, 0, \frac{1}{2}\right)$, employing four integers $(h k l m)$ (see Figure 2 (a),(b)). The fourfold superstructure features rows of three satellite reflections between main reflections. Thus, reflection $(h k l m)$ may be equally indexed as $(h+1 k l+2 m-4)$, and for a unique indexing, the range of $m$ values should be restricted to $-1,0,1,2$. The conventional indexing with three indices would require a primitive monoclinic unit cell as shown on Figure 2(c). Finally, the structure may be described with a non-standard $4 a \times b \times 2 c B$-centered supercell (Figure 2(d)).

The diffraction pattern of $\mathrm{CrOCl}$ at $22.9 \mathrm{GPa}$ is more complex. It can be indexed with the unit cell of the basic structure and $\boldsymbol{q}^{C r}=\left(\frac{2}{7}, 0, \frac{1}{2}\right)$ or with a 14-fold orthorhombic supercell (Figure 3).

For any commensurately modulated structure, data integration may be performed on the basis of the supercell indexing. Later the supercell-superspace indices transformation can be performed during data importing into JANA2006. Data integration and reduction were performed with CrysAlisPro program suite which contains useful features for high-pressure data measured in a DAC [28, 29]. For example, it is possible to introduce the DAC opening angle for the correct treatment of DAC shadowing. The built-in explorer of the reciprocal space simplifies the process of the unit cell finding. Furthermore, 

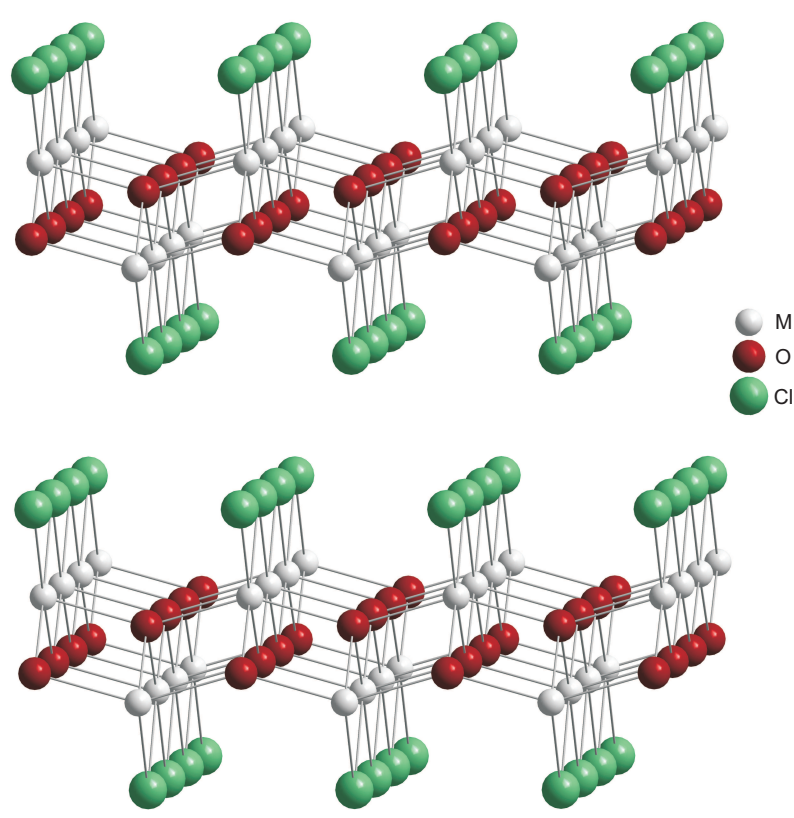

Figure 1. Crystal structure of $\mathrm{MOCl}$ at ambient conditions.

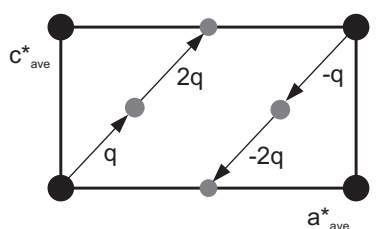

(a)

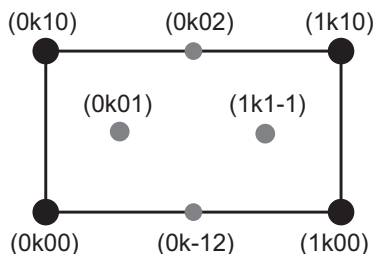

(b)

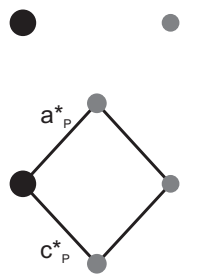

-

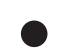<smiles>C1O[C@@H]2C[C@H](O1)O2</smiles>

\section{0}<smiles>C1CCC1</smiles>

$\bigcirc$

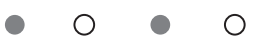

(c)

(d)

Figure 2. Different ways of indexing. Shown are reciprocal lattice planes perpendicular to $\mathbf{b}^{*}$. (a),(b) - Schematic depiction of the diffraction pattern of $\mathrm{FeOCl}$ with main and satellite reflections depicted as black and grey circles respectively, and modulation wavevector $\boldsymbol{q}=\frac{1}{4} \boldsymbol{a}^{*}+\frac{1}{2} \boldsymbol{c}^{*}$. (c) Indexing by a monoclinic primitive unit cell (d) Indexing with pseudoorthorhombic non-conventional $B$-centered $4 a \times 1 b \times 2 c$ supercell.

CrysAlisPro is compatible with several instruments and detector types. The in-house software, which allows to convert images to required formats is available at beamlines P02.2 and ID09A. In case of incommensurate structures one can use built-in procedure $N A D A$ for the simultaneous refinement of the orientation matrix and modulation vector [30.

Special care must be taken for the correct rejection of outlying reflections because standard procedures that work well for conventional data sets, may fail to recognize a significant number of outliers in case of high-pressure data. In the last years several procedures for outlier identification during the structure determination process were 


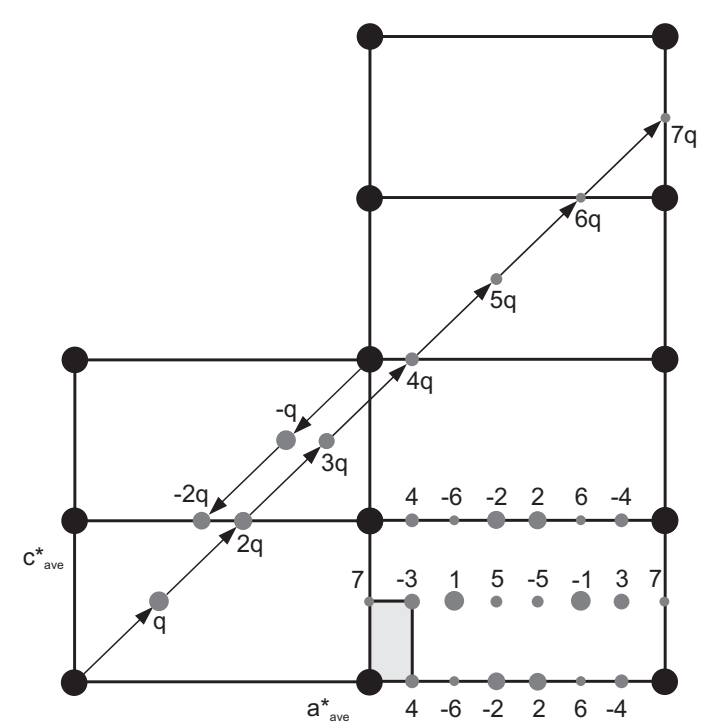

Figure 3. Schematic drawing of the diffraction pattern of $\mathrm{CrOCl}$ at $22.9 \mathrm{GPa}$. Only selected superlattice reflections are indicated. The indexing with multiples of the modulation wavevector $q=\frac{2}{7} a^{*}+\frac{1}{2} c^{*}$ is demonstrated. The right-lower reciprocal unit cell shows all superlattice reflections of orders $m=-6$.. 7 . The volume of the superstructure unit cell is 14 times larger than that of the unit cell of the average structure. The reciprocal 3D supercell is indicated.

implemented into JANA2006 program [31].

\section{$2.3 \quad$ Structure solution and refinement}

The first step in the process of the structure solution is the determination of the symmetry. Usually, the superspace groups compatible with the symmetry of the data are proposed by JANA2006. In the present case this leads to two possible superspace groups: $\operatorname{Pmmn}\left(\sigma_{1} 0 \frac{1}{2}\right) 00 s$ and $\operatorname{Pmmn}\left(\sigma_{1} 0 \frac{1}{2}\right) 000$, which in fact are the different settings of Pmnm $\left(0 \frac{1}{2} \sigma_{3}\right) 000$ (No. 59.1.10.6) [32, 33]. So, all possibilities must be probed for the structure solution. For both $\mathrm{CrOCl}$ and $\mathrm{FeOCl} P m m n\left(\sigma_{1} 0 \frac{1}{2}\right) 00 s$ appeared to be the true superspace symmetry.

The superspace analysis immediately shows that the high-pressure phases of $\mathrm{CrOCl}$ and $\mathrm{FeOCl}$ can be obtained as different distorted variants of the ambient-pressure phase. In principal, when the crystal structure of the non-distorted phase is known, the $a b$ initio solution of the superstructure is not required, because atomic coordinates in the parent structure can be used as a starting point for the superstructure refinements. In general, a fourfold superstructure (in case of $\mathrm{FeOCl}$ ) would require only up to secondorder harmonics for a complete description. However, non-trivial point symmetry requires higher-order harmonics [12]. Thus, the value of $n_{\max }$ (see Eq. 3) was determined for every atom individually. One must take care that introduction of higher-order harmonics is justified by a significant drop in the agreement factors and that their refined values are meaningful.

For a commensurate modulation, one superspace group corresponds to different 3D structures. Accordingly, different sections $t=t_{0}$ of superspace lead to different symmetries of the $4 a \times b \times 2 c$ supercell in $3 \mathrm{D}$ space. They are $B m, B 2_{1} / m$ (both are $b$-unique) and $B m m 2$. The best fit to the diffraction data was achieved using $t_{0}=\frac{1}{16}+\frac{N}{4}(N=0,1,2,3)$, which corresponds to the centrosymmetric, monoclinic space group $B 2_{1} / m$ (No. 11 with standard setting $\left.P 2_{1} / m\right)$ [34].

Employing the superspace approach, we consider the basic structure of FeOCl containing only three independent atoms on the $m m 2$ positions. Symmetry restrictions on 


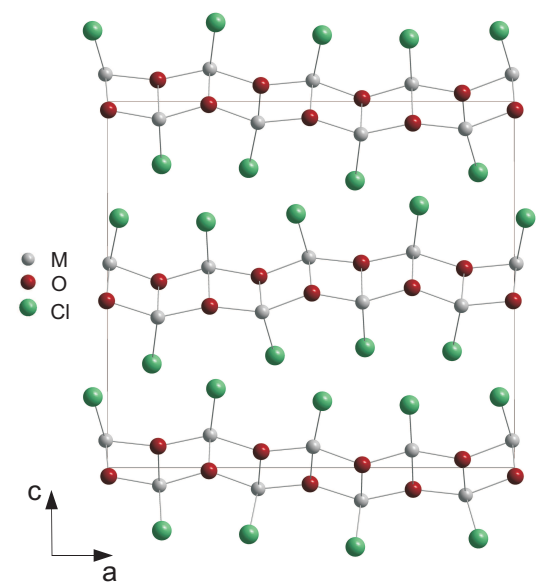

(a)

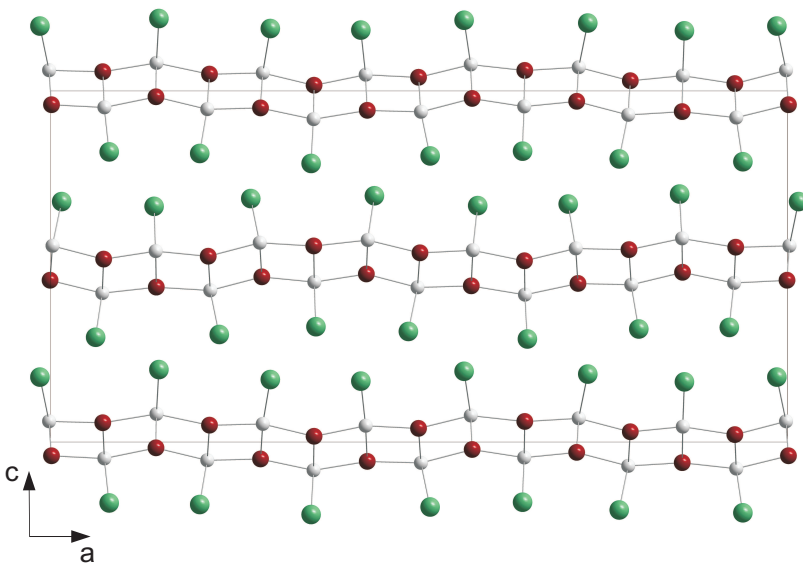

(b)

Figure 4. Superstructures of $\mathrm{FeOCl}(\mathrm{a})$ and $\mathrm{CrOCl}$ (b) at $23 \mathrm{GPa}$.

the modulation functions lead to 14 independent amplitudes resulting in 17 positional parameters to be refined. 19 more parameters were used for the atomic displacement parameters (ADPs). As a result, together with scale factor and the twin volume ratio, 38 parameters have been refined (Table 1). At the same time the conventional refinement in the supercell failed with 62 refinable parameters.

In case of $\mathrm{CrOCl}$ the situation is slightly different. The 14 -fold superstructure would allow satellites up to $7^{\text {th }}$ order, however only first and second order satellites were observed. As a consequence it is impossible to distinguish between incommensurate and commensurate modulations, and between structures with different values of $t_{0}$. The possible symmetries of superstructures are Pcmn for $t_{0}=0+\frac{N}{14}, P m m n$ for $t_{0}=\frac{1}{28}+\frac{N}{14}$ and $P 2{ }_{1} m n$ for general $t$-section. According to the structure refinement Pcmn, Pmmn and incommensurate models have the same agreement factors (Table 1). In this case the determination of the true structure and its symmetry is impossible, but the treatment of the structure as an incommensurate may serve as a good approximation to the real structure. Thus, there are not just 14 specific values for e.g. interatomic distances between two atoms, but a range of values with a minimum, a maximum and an average value. Since atomic modulation functions include harmonics only up to second order and do not reveal local features, these 14 values perfectly define the curve and cover the same range (Figure 5). As a consequence, the crystal-chemical analysis doesn't suffer much from this uncertainty. The structure of $\mathrm{CrOCl}$ can be described with only 23 structural parameters, while 106 parameters are required for 3D superstructure models.

\subsection{Crystal-chemical analysis}

The superstructures of $\mathrm{FeOCl}$ and $\mathrm{CrOCl}$ are presented on Figure 4 . Both structures reveal the antiphase buckling of $M-\mathrm{O}$ bilayers with pronounced variation of interlayer distances. For the crystal-chemical analysis of superstructures so-called $t$-plots may be used. They represent the variation of interatomic distances, bond angles, bond valences or the values of the modulation functions themselves with the phase of modulation. Furthermore, it is important to study the correlations between $t$-plots of different quantities.

Selected $t$-plots are presented on the Figure 6. For FeOCl structural distortions lead to the formation of the fourfold superstructure and resulted in the appearance of four crystallographic sites for each atom, thus there are four points on the $t$-plots corresponding to the real structure indicated by vertical dashed lines. The largest displacements of Fe and $\mathrm{O}$ atoms were found along $\boldsymbol{z}$ (Figure 6(a)). These displacements define the buckling 


\begin{tabular}{lcc}
\hline & FeOCl & $\mathrm{CrOCl}$ \\
\hline Number of parameters refined & 38 & 23 \\
R $_{\text {int } \text { (obs/all) }}$ & $5.00 / 5.15$ & $6.65 / 6.74$ \\
No. of reflections & & \\
(measured/unique): & & \\
All & $966 / 404$ & $724 / 231$ \\
Main & $241 / 101$ & $140 / 44$ \\
$1^{\text {st } \text { order satellites }}$ & $484 / 201$ & $331 / 96$ \\
$2^{\text {nd } \text { order satellites }}$ & $241 / 102$ & $253 / 91$ \\
No. of observed reflections & & \\
(all/unique): & & \\
All & $802 / 311$ & $549 / 154$ \\
Main & $211 / 84$ & $126 / 39$ \\
$1^{\text {st } \text { order satellites }}$ & $393 / 155$ & $259 / 65$ \\
$2^{\text {nd } \text { order satellites }}$ & $198 / 72$ & $164 / 50$ \\
Robs $/$ Rall: & & \\
All & $5.29 / 6.95$ & $4.08 / 5.94$ \\
Main & $4.00 / 4.60$ & $3.69 / 3.94$ \\
$1^{\text {st } \text { order satellites }}$ & $6.93 / 8.68$ & $4.45 / 6.77$ \\
$2^{\text {nd } \text { order satellites }}$ & $5.41 / 9.68$ & $4.31 / 8.65$ \\
\hline
\end{tabular}

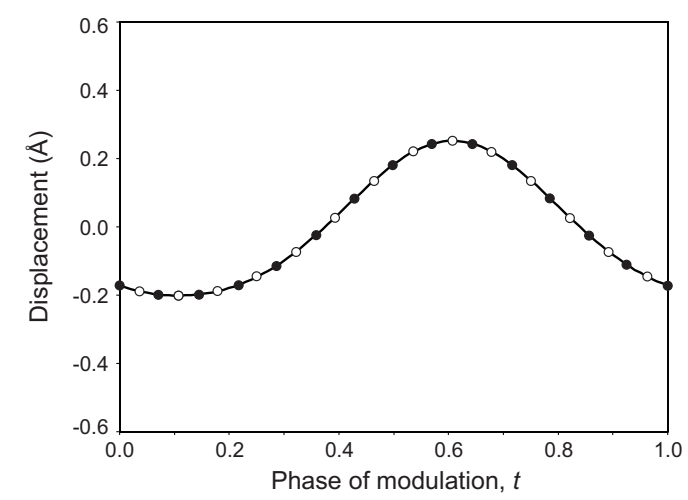

Figure 5. $\quad t$-plot for $z$-displacement of $\mathrm{Cl}$ atom in $\mathrm{CrOCl}$ at $22.9 \mathrm{GPa}$. Filled and opened circles represent values for commensurate Pcmn and Pmmn models respectively.

of $\mathrm{Fe}-\mathrm{O}$ layers. It could be noticed that the $\mathrm{Cl}$ atom perfectly follows the displacements of Fe. The $\mathrm{O}$ atom has comparable displacement amplitudes, but a different phase.

$\mathrm{Fe}$ and $\mathrm{O}$ atoms don't possess large displacements along $\boldsymbol{x}$ due to rigidity of the layers, but the $\mathrm{Cl}$ atom is relatively flexible within the interlayer gap (Figure 6(b)). While the magnitudes of atomic displacements are about $1 \AA$, they are correlated in a way to prevent very unfavorable short bond lengths (Figure 6(c)-(f)). The largest variation of bond length found is $\sim 0.08 \AA$ for Fe-O bond along $\boldsymbol{y}$, which is at its longer limit at ambient pressure and therefore has more freedom. Exactly the same considerations are valid for the description of the $\mathrm{CrOCl}$ structure (Figure 6(g)-(l)). Actually, the superstructures of $\mathrm{FeOCl}$ and $\mathrm{CrOCl}$ differ only in the period of the modulation, but have similar modulation functions and can be described by the same superspace group, since the distortion mechanism is the same.

A detailed study of $t$-plots of $\mathrm{CrOCl}$ and $\mathrm{FeOCl}$ towards understanding the mechanisms of the phase transitions will be given elsewhere.

\section{Conclusions}

We have demonstrated that the application of the superspace approach is useful in the analysis of high-pressure superstructures. Firstly, it helps to significantly reduce the num- 


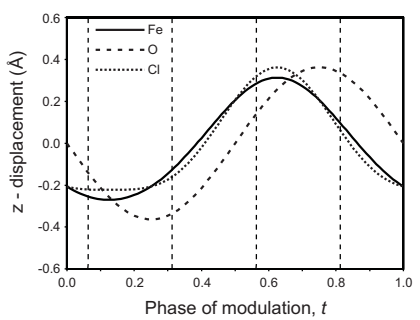

(a)

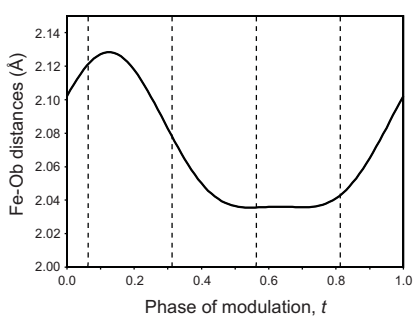

(d)

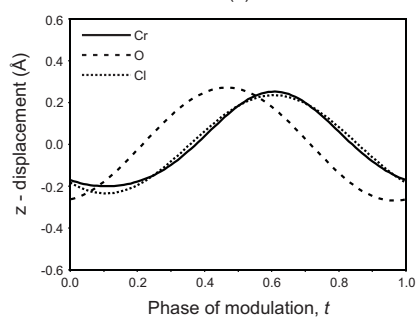

(g)

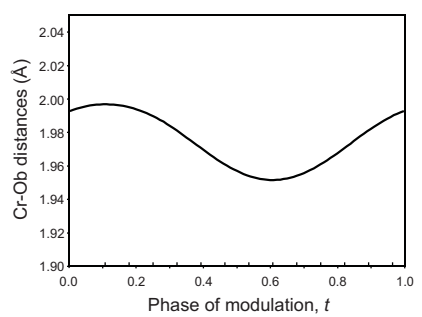

(j)
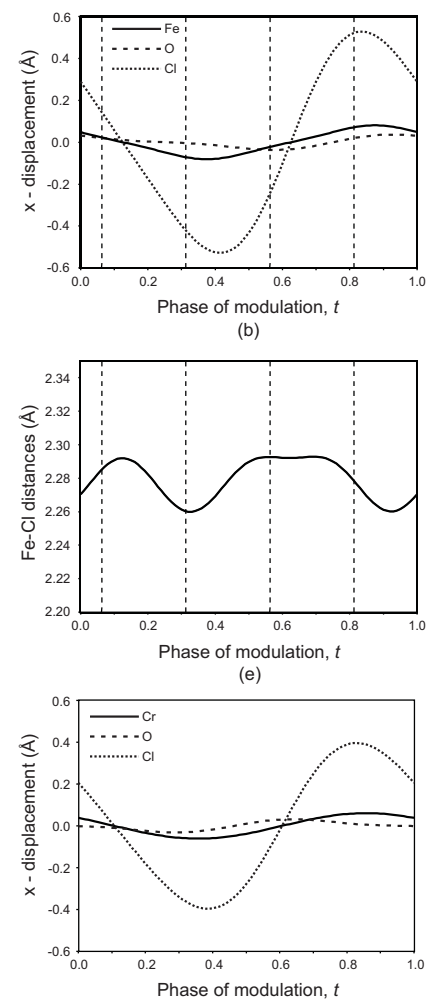

(h)

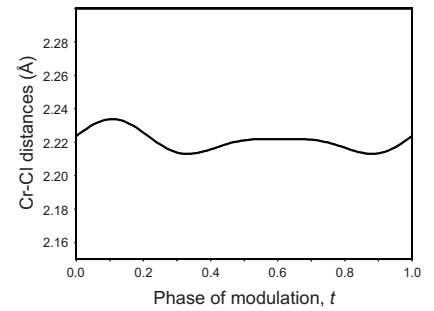

(k)

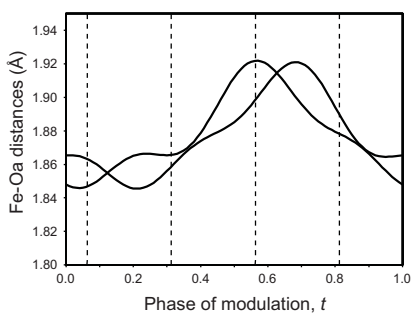

(c)

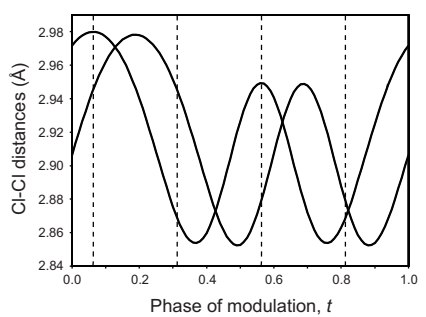

(f)

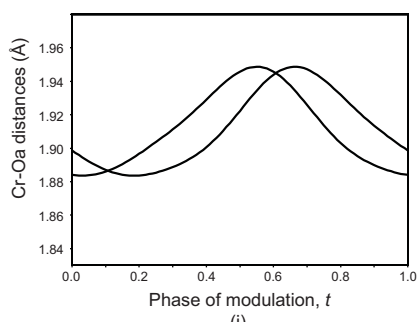

(i)

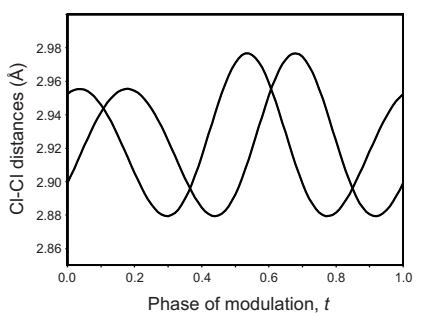

(I)

Figure 6. $t$-plots for selected interatomic distances and atomic displacements for $\mathrm{FeOCl}$ (a)-(f) and for $\mathrm{CrOCl}$ (g)-(l). Vertical dashed lines indicate commensurate $t$-sections. For further explanations see text.

ber of refined structural parameters and to get a reliable structural model in cases when conventional structure refinement fails. Secondly, superspace tools provide an elegant way of crystal-chemical analysis. It provides a tool to study direct relations between the parent and distorted phases as well as between distorted phases with different modulation periods.

\section{Acknowledgements}

We thank Prof. Dr. Leonid Dubrovinsky for critical reading of the manuscript and for the help with synchrotron experiments. We thank Alfred Suttner for growing single crystals of FeOCl and $\mathrm{CrOCl}$. Financial support by the German Science Foundation (DFG) is gratefully acknowledged.

\section{References}

[1] Bassett WA. Diamond anvil cell, 50th birthday. High Press. Res. 2009;29:163-186. 
[2] McMahon MI. High-pressure crystallography. In: Rissanen K. Advanced X-ray crystallography: Springer-verlag Berlin;2012. p. 69-109.

[3] Pasternak MP, Taylor RD, Chen A, Meade C, Falicov L, Giesekus A, Jeanloz R, Yu PY. Pressure-induced metallization and the collapse of the magnetic state in the antiferromagnetic insulator $\mathrm{NiI}_{2}$. Phys. Rev. Lett. 1990;65:790-793.

[4] Patterson J, Aracne C, Jackson D, Malba V, Weir S, Baker P, Vohra Y. Pressure-induced metallization of the Mott insulator MnO. Phys. Rev. B. 2004;69:220101(R).

[5] Takano M, Nasu S, Abe T, Yamamoto K, Endo S, Takeda Y, Goodenough J. Pressureinduced high-spin to low-spin transition in $\mathrm{CaFeO}_{3}$. Phys. Rev. Lett. 1991;67:3267-3270.

[6] Chen XJ, Struzhkin VV, Yu Y, Goncharov AF, Lin CT, Mao HK, Hemley RJ. Enhancement of superconductivity by pressure-driven competition in electronic order. Nature. 2010;466:950-953.

[7] McMahon MI, Nelmes RJ. High-pressure structures and phase transformations in elemental metals. Chem. Soc. Rev. 2006;35:943-963.

[8] Manjón FJ, Errandonea D. Pressure-induced structural phase transitions in materials and earth sciences. Phys. Status Solidi B.2009;246:9-31.

[9] Dera P, Nisar J, Ahuja R, Tkachev S, Prakapenka VB. New type of possible high-pressure polymorphism in NiAs minerals in planetary cores. Phys. Chem. Miner. 2012;40:183-193.

[10] Sowa H, Steurer W, de Boer JL. Pressure-induced phase transitions in $\mathrm{Mo}_{8} \mathrm{O}_{23}$. Phase Transitions. 1994;47:1-8.

[11] Palmer D, Finger L. Pressure-induced phase transition in cristobalite: an X-ray powder diffraction study to 4.4 GPa. Am. Mineral. 1996;79:1-8.

[12] van Smaalen S. Incommensurate crystallography. Oxford University Press; 2007.

[13] Janssen T, Ghapuis G, de Boissieu M. Aperiodic Crystals. From Modulated Phases to Quasicrystals. Oxford University Press; 2007.

[14] Wagner T, Schönleber A. A non-mathematical introduction to the superspace description of modulated structures. Acta Crystallogr. B. 2009;65:249-268.

[15] Palatinus L, Chapuis G. SUPERFLIP - a computer program for the solution of crystal structures by charge flipping in arbitrary dimensions. J. Appl. Crystallogr. 2007;40:786-790.

[16] Petricek V, Dusek M, Palatinus L. Jana2006. The crystallographic computing system. Institute of Physics, Praha, Czech Republic. 2006.

[17] Schreurs AMM, Xian X, Kroon-Batenburg LMJ. EVAL15 : a diffraction data integration method based on ab initio predicted profiles. J. Appl. Crystallog. 2009;43:70-82.

[18] Perez-Mato JM, Elcoro L, Aroyo MI, Katzke H, Tolédano P, Izaola Z. Apparently Complex High-Pressure Phase of Gallium as a Simple Modulated Structure. Phys. Rev. Lett. 2006;97:115501.

[19] Perez-Mato JM, Orobengoa D, Aroyo MI. Mode crystallography of distorted structures. Acta Crystalogr. A. 2010;66:558-590.

[20] Kantor I, Prakapenka VB, Kantor A, Dera P, Kurnosov A, Sinogeikin S, Dubrovinskaia N, Dubrovinsky L. BX90: a new diamond anvil cell design for X-ray diffraction and optical measurements. Rev. Sci. Instrum. 2012;83:125102.

[21] Boehler R, De Hantsetters K. New anvil designs in diamond-cells. High Press. Res. 2004;24:391-396.

[22] Mao HK, Xu J, Bell PM. Calibration of the ruby pressure gauge to 800 kbar under quasihydrostatic conditions. J. Geophys. Res. 1986;91:4673.

[23] Kurnosov A, Kantor I, Boffa-Ballaran T, Lindhardt S, Dubrovinsky L, Kuznetsov A, Zehnder BH. A novel gas-loading system for mechanically closing of various types of diamond anvil cells. Rev. Sci. Instrum. 2008;79:045110.

[24] Seidel A, Marianetti C, Chou C, Ceder G, Lee P. S=1/2 chains and spin-Peierls transition in TiOCl. Phys. Rev. B.2003;67:020405(R).

[25] Schönleber A, Angelkort J, van Smaalen S, Palatinus L, Senyshyn A, Morgenroth W. Phase transition, crystal structure, and magnetic order in VOCl. Phys. Rev. B. 2009;80:064426.

[26] Angelkort J, Wölfel A, Schönleber A, van Smaalen S, Kremer RK. Observation of strong magnetoelastic coupling in a first-order phase transition of CrOCl. Phys. Rev. B. 2009;80:144416.

[27] Zhang J, Wölfel A, Li L, van Smaalen S, Williamson H, Kremer RK. Magnetoelastic coupling in the incommensurate antiferromagnetic phase of FeOCl. Phys. Rev. B. 2012;86:134428. 
[28] Xcalibur CCD system. CrysAlisPro Software system. Version 1.171.34. Agilent Technologies; 2010.

[29] Xcalibur CCD system. CrysAlisPro Software system. Version 1.171.32. Oxford Diffraction; 2007.

[30] Schönleber A, Meyer M, Chapuis G. NADA - a computer program for the simultaneous refinement of orientation matrix and modulation vector(s). J. Appl. Crystallogr. 2001;34:777779.

[31] Friese K, Grzechnik A, Posse JM, Petricek V. Refinement of high pressure single-crystal diffraction data using Jana2006. High Press. Res. 2013;33:196-201.

[32] Stokes HT, Campbell BJ, van Smaalen S. Generation of $(3+\mathrm{d})$-dimensional superspace groups for describing the symmetry of modulated crystalline structures. Acta Crystallogr. A. 2011;67:45-55.

[33] van Smaalen S, Campbell BJ, Stokes HT. Equivalence of superspace groups. Acta Crystallogr. A. 2013;69:75-90.

[34] Hahn T. International Tables for Crystallography Volume A: Space-group symmetry. Springer Netherlands; 2002. 